\title{
Estrategias de aprendizaje organizacional y tecnologías de la información y las comunicaciones para apoyar la gestión de conocimiento en las pymes del Valle del Cauca, Colombia
}

\author{
Adriana Patricia Bermúdez-Arango ${ }^{1}$ \\ Universidad del Valle \\ bermudez.adriana@correounivalle.edu.co \\ Carlos Julián Cuéllar-Torres ${ }^{2}$ \\ Universidad del Valle \\ cuellar.carlos@correounivalle.edu.co \\ Sandra Cristina Riascos-Erazo ${ }^{3}$ \\ Universidad del Valle \\ sandra.riascos@correounivalle.edu.co
}

Fecha de recepción: 11 de septiembre de 2019

Fecha de aprobación: 11 de noviembre de 2020

Fecha de publicación: 18 de marzo de 2021

Cómo citar este artículo / To reference this article / Comment citer cet article / Para citar este artigo:

Bermúdez-Arango, A. P.; Cuéllar-Torres, C. J.; Riascos-Erazo, S. C. (2020). Estrategias de aprendizaje organizacional y tecnologías de la información y las comunicaciones para apoyar la gestión de conocimiento en las pymes del Valle del Cauca, Colombia. Revista Escuela de Administración de Negocios, (89), 69-90. DOI: https://doi.org/10.21158/01208160.n89.2020.2818

\section{Resumen}

La evolución en la sociedad ha conllevado a la consolidación de la economía del conocimiento, en la que las pymes se enfrentan al desafío de gestionar de forma adecuada el know how de la organización, para lo cual existen diversas prácticas organizacionales que facilitan este proceso. Teniendo en cuenta lo anterior, el artículo tiene como objetivo proponer una serie de estrategias que les permitan a las pymes fortalecer la gestión del conocimiento desde su relación con el aprendizaje organizacional y las tecnologías de la información y las comunicaciones (TIC). Este estudio se desarrolló a través de la aplicación de una encuesta estructurada con preguntas cerradas, dirigidas a los gerentes o líderes de 117 pymes del Valle del Cauca. Entre los principales resultados de la investigación se evidencia la carencia de un área formal para la gestión del conocimiento, y que las pymes cuentan con procesos colaborativos que facilitan compartir el conocimiento entre los miembros de la organización. Sin embargo, no se han establecido mecanismos formales e institucionales para la transferencia de conocimiento que permita una adecuada documentación y su correcto almacenamiento. Así mismo, existe una debilidad en la falta de cultura organizacional que

Maestría en Administración de la Universidad del Valle. ORCID: https://orcid.org/0000-0002-7433-5706

Maestría en Administración de la Universidad del Valle. ORCID: https://orcid.org/0000-0002-3438-7173

3 Doctora en Ingeniería Informática de la Universidad Carlos III de Madrid. Auditora de Sistemas de la Universidad Antonio Nariño y Licenciada en Informática de la Universidad de Nariño. Directora del Grupo de Investigación en Inteligencia de Negocios e Ingeniería del Conocimiento. ORCID: https://orcid.org/0000-0002-4595-1737 
fomente y facilite este intercambio de conocimiento, por lo cual se proponen algunas estrategias, como, por ejemplo, mejorar los mecanismos y las prácticas de transferencia y la implementación de plataformas virtuales de aprendizaje, entre otras.

Palabras clave: gestión del conocimiento; transferencia del conocimiento; intercambio de conocimiento; aprendizaje organizacional; cultura organizacional; pymes; tecnologías de la información y la comunicación.

\title{
Organizational learning strategies and information and communication technologies to support knowledge management in SMEs in Valle del Cauca, Colombia
}

\begin{abstract}
The evolution of society has led to the consolidation of a knowledge economy where SMEs have to face the challenge of adequately managing the know-how of organizations, but there are also several organizational practices that facilitate this process. In view of the above, this article aims at proposing a series of strategies that allow SMEs to strengthen knowledge management from their relationship with organizational learning and Information and Communication Technologies (ICT). This study is developed through a survey that was structured with closed questions, addressed to managers or leaders of 117 SMEs in Valle del Cauca. Among the main research findings, it is clear that there is no formal area for knowledge management, and that the SMEs rely on collaborative processes that facilitate sharing knowledge among the members of the organization. However, formal and institutional mechanisms for transferring knowledge that would allow adequate documentation and its correct storage have not been established. There is also a weakness in the lack of an organizational culture that encourages and facilitates this exchange of knowledge, reason for this document to propose some strategies, such as improving the mechanisms and practices for knowledge transfer and the implementation of virtual learning platforms, among others.
\end{abstract}

Keywords: knowledge management; knowledge transfer; knowledge exchange; organizational learning; organizational culture; SMEs; information and communication technologies.

\section{Estratégias de aprendizagem organizacional e tecnologias de informação e comunicação para apoiar a gestão do conhecimento em PME no Valle del Cauca, Colômbia}

\section{Resumo}

A evolução da sociedade tem conduzido à consolidação da economia do conhecimento, na qual as PME enfrentam o desafio de administrar de forma adequada o know-how (saber-como) da organização, na qual existem várias práticas organizacionais que facilitam esse processo. Tendo isso em consideração, o artigo tem como objetivo propor um conjunto de estratégias que permitem às PME reforçarem a gestão do conhecimento a partir da sua relação com a aprendizagem organizacional e as tecnologias de informação e comunicação (TIC). Este estudo foi desenvolvido através da aplicação de um questionário estruturado com perguntas fechadas, dirigido aos gerentes ou líderes de 117 PME do Vale do Cauca. Entre os principais resultados da pesquisa está a falta de uma área formal para a gestão do conhecimento, e que as PME possuem processos colaborativos que facilitam o compartilhamento do conhecimento entre os membros da organização. No entanto, não foram estabelecidos mecanismos formais e institucionais para a transferência 
de conhecimentos que permitam uma documentação adequada e seu correto armazenamento. Mesmo assim, existe uma fragilidade na falta de uma cultura organizacional que incentive e facilite essa troca de conhecimentos, para a qual algumas estratégias são propostas, como o aprimoramento dos mecanismos e práticas de transferência e a implantação de plataformas virtuais de aprendizagem, entre outras.

Palavras-chave: gestão do conhecimento; transferência de conhecimento; troca de conhecimento; aprendizado organizacional; cultura organizacional; PME; Tecnologia de informação e comunicação.

\section{Stratégies d'apprentissage organisationnel et technologies de l'information et de la communication pour l'aide à la gestion des savoir-faire des $\square$ PME du Valle del Cauca, Colombie}

\section{Résumé}

L'évolution de la société a conduit à une consolidation de l'économie de la connaissance où les PME sont confrontées au défi d'une gestion efficiente du savoir-faire entrepreneurial que peuvent faciliter différentes pratiques organisationnelles. Cet article propose une série de stratégies permettant aux PME de renforcer la gestion de leurs savoir-faire via l'apprentissage organisationnel et les technologies de l'information et de la communication (TIC). Létude a été réalisée au travers d'une enquête structurée de questions fermées adressées aux dirigeants de 117 PME de la région du Valle del Cauca, en Colombie. Parmi les principaux résultats de cette étude figurent l'absence de section formelle chargée de la gestion des savoir-faire pour en faciliter le partage entre les membres de l'entreprise. Aucun mécanisme formel n'a jusqu'ici été mis en place permettant le transfert des connaissances, leur utilisation et leur stockage adéquate. L'étude montre par ailleurs une culture organisationnelle encourageant et facilitant l'échange des savoir-faire grâce, entre autres, à une amélioration des mécanismes et des pratiques de transfert ou via la mise en place de plateformes d'apprentissage virtuelles.

Mots-clés: gestion des savoir-faire; transfert des connaissances; échange des connaissances; apprentissage organisationnel; culture organisationnelle; PME; technologie de l'information et de la communication. 


\section{Introducción}

$\mathrm{E}$ Colombia se destaca que las micro, pequeñas y medianas empresas representan el $96 \%$ del tejido empresarial, aportan el $40 \%$ al PIB, generan más de diecisiete millones de empleos y representan el 9,8\% de las exportaciones nacionales (Quintero, 2019). No obstante, según Pérez-Uribe y Ramírez (9 de febrero de 2015), existen aspectos importantes relacionados con el fracaso de las pymes en Colombia, referidos a la poca importancia que se le da a la innovación y al conocimiento. Por tanto, se constituye en una dificultad que se debe atender mediante procesos investigativos que tengan como objetivo evidenciar las actividades desarrolladas dentro de las pymes, a fin de presentar propuestas de mejoramiento relacionadas con la gestión del conocimiento que conlleven a la innovación.

Por otra parte, las pymes del Valle del Cauca carecen de estudios documentados que permitan orientar las dinámicas de aprendizaje organizacional y las TIC que contribuyan a la gestión del conocimiento. En este sentido, este estudio recolectó información importante que permitió construir estrategias fundamentadas sobre las vivencias, las expectativas y las necesidades que tienen actualmente las pymes.

Abordar temáticas tales como el aprendizaje organizacional y las TIC que presentan avances interesantes tanto para el progreso de las organizaciones como desde su aporte a la competitividad y la innovación (Bibi et al., 2020; Bilan, Hussain, Haseeb y Kot, 2020; Hindasah y Nuryakin, 2020) justifica la importancia del estudio en esta temática. Además, a partir de esta investigación es posible proponer estrategias de apoyo para que las pymes se constituyan en organizaciones más productivas.

La importancia de la gestión del conocimiento en las pymes - tanto para su desarrollo organizacional como para su sostenibilidad en un contexto económico de gran competitividad - ha sido tema de estudio de diferentes autores, entre los que se destacan Senge (1999), desde la iniciativa de una organización inteligente; Berawi (2004), en su relación con la gestión de la calidad; y Papa et al. (2018), con el aporte de la gestión del conocimiento para la innovación, entre otros autores.

La investigación se planteó como objetivo proponer una serie de estrategias que permitan fortalecer la gestión del conocimiento desde su relación con el aprendizaje organizacional y las tecnologías de la información y las comunicaciones (TIC), a partir del reconocimiento de la dinámica de las pymes del Valle del Cauca en el tema de la gestión del conocimiento desde la perspectiva tecnológica y del aprendizaje organizacional.

El estudio se desarrolló desde un enfoque cuantitativo y utilizó un cuestionario aplicado a 117 pymes, lo que corresponde a la muestra de 7903 pymes registradas en la Cámara de Comercio de Cali en el 2017 (20 de agosto de 2019). La información obtenida se analizó en el software SPSS y la investigación limitó el análisis a empresas ubicadas en el casco urbano de Cali, así como a empresas que tienen por lo menos diez años de constituidas.

Este artículo presenta cinco apartados: un referente teórico, en el que se describen algunos elementos teóricos y conceptuales que permitieron fundamentar el análisis y los resultados de la investigación; la metodología, en la que se exponen los aspectos de recolección y análisis de información; los resultados y la discusión, en los que se ilustran los principales hallazgos que se resaltan luego del análisis de la información, así como la contribución del estudio en la propuesta de una serie de estrategias que permitirían a las pymes aprovechar las bondades de las TIC y el aprendizaje organizacional para potencializar la gestión del conocimiento; finaliza con las conclusiones, en las que se expone el aporte del estudio al área de conocimiento. 


\section{Referente teórico}

$\mathrm{D}$ e acuerdo con Muñoz y Riverola (2003), el conocimiento se define como la capacidad de resolver un conjunto determinado de problemas. Complementan Alavi y Leidner (2003) al afirmar que el conocimiento es la información que el individuo posee en su mente, personalizada y subjetiva, relacionada con hechos, procedimientos y conceptos, así como elementos que pueden ser útiles, precisos o estructurales. Por otra parte, la RAE (2018) define conocimiento como «entendimiento», «inteligencia», «razón natural». El conocimiento se ha constituido en un recurso económico dominante que genera competitividad (Drucker,1999); en este sentido, se origina la gestión del conocimiento que tiene sus cimientos en Amitai Etzioni, en su libro Organizaciones modernas (1964), en el cual afirma que todas las unidades sociales utilizan el conocimiento, pero son las organizaciones las que usan más conocimientos y de manera más sistémica.

Para los fines de la investigación, la gestión del conocimiento se entiende como el conjunto de prácticas, procedimientos y técnicas que le permiten a las organizaciones: a) identificar los conocimientos requeridos para realizar sus actividades presentes $\mathrm{y}$ futuras, b) disponer de dichos conocimientos $y$ c) aplicarlos de forma adecuada a fin de lograr los resultados esperados (Davenport y Prusak, 2001; Koulopoulos y Frappaolo, 2001; Nie, Ma y Nakamori, 2009).

En los últimos años se ha evidenciado que la gestión del conocimiento tiene un impacto significativo en el desempeño organizacional, tal como lo argumentan Ahmad, Lodhi, Zaman y Naseem (2017), Ferraris et al. (2017), y Vrontis, Thrassou, Santoro y Papa (2017); en este sentido, la gestión del conocimiento eficiente permite que las organizaciones puedan realizar más actividades innovadoras, mejorar su rendimiento e incrementar su competitividad en el sector.

Entre las teorías en este campo se encuentra también el planteamiento referido al ciclo de la gestión del conocimiento, en el cual se involucran, por lo general, conceptos tales como captura, identificación, adquisición, generación y difusión del conocimiento en la organización; Dalkir y Liebowitz (2011) exponen un ciclo de gestión del conocimiento en el que se destacan tres conceptos: captura o creación de conocimiento, compartir o diseminar conocimiento $y$, finalmente, la adquisición y su aplicación en el contexto (véase la Figura 1).

Figura 1. Ciclo de la gestión del conocimiento

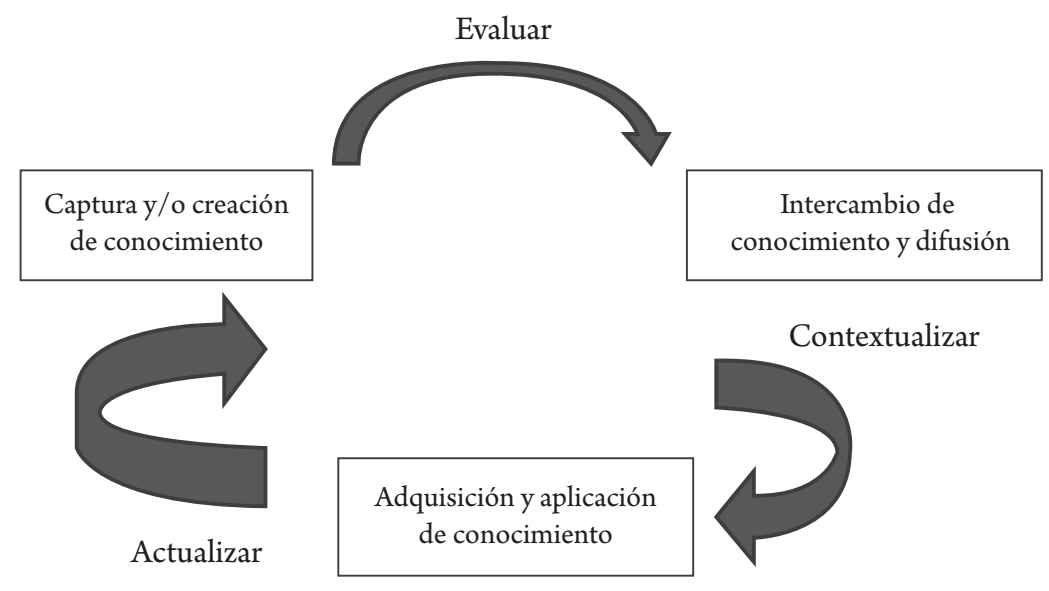

Fuente. Dalkir y Liebowitz, 2011. 
Dentro de este proceso de la gestión del conocimiento existe una fuerte relación entre las organizaciones y el desarrollo de las TIC, la cual abordan QuirogaParra et al. (2010). En esta se evidencia que, a través de la historia, el aprendizaje ha estado apoyado por la tecnología, y ha sido precisamente el desarrollo de esta última lo que ha permitido la creación de redes que facilitan la transmisión de conocimiento, el cual es responsable, a su vez, de generar organizaciones más productivas al capacitar de manera adecuada a su personal.

\subsection{Aprendizaje organizacional}

Para hablar de aprendizaje organizacional se debe comenzar por analizar el aprendizaje en sí mismo, esa necesidad que se tiene, al vivir en una sociedad, de conocer lo que en ella ocurre y participar de manera activa en este conocimiento. Tal como lo manifestó Escudero-Yerena (1998), el aprendizaje es un proceso de toda la vida, debido a que consta de varias etapas $y$, en cada una de ellas, el ser humano desarrolla sus capacidades naturales. Estas se aplican cuando, incluso de manera intrínseca, el ser humano comparte lo aprendido al enseñar a otros.

En suma, la tabla 1 ilustra la recopilación de los conceptos de aprendizaje organizacional con mayor relevancia y que permiten tener mayor claridad del tema de investigación.

Tabla 1. Aprendizaje organizacional según varios autores

\begin{tabular}{|c|c|}
\hline Autor/es & Definición \\
\hline Fiol y Lyles (1985) & $\begin{array}{l}\text { Proceso a través del cual se emplean el conocimiento y el entendimiento con el objetivo de } \\
\text { mejorar las acciones, y en el cual la experiencia de los miembros de la organización juega un papel } \\
\text { fundamental en el desarrollo de nuevos conocimientos que influencian de manera positiva la } \\
\text { organización. }\end{array}$ \\
\hline Nonaka y Takeuchi (1995) & $\begin{array}{l}\text { Proceso por el cual se amplifica de forma organizada el conocimiento creado por los individuos y se } \\
\text { cristaliza como parte del sistema de conocimientos de la organización. }\end{array}$ \\
\hline Kluge y Schilling (2000) & $\begin{array}{l}\text { Aprendizaje que surge de forma cooperativa dentro de un sistema social y, a su vez, la organización } \\
\text { que aprende se define como el marco formal que permite dar continuidad y sostenibilidad a dicho } \\
\text { aprendizaje. }\end{array}$ \\
\hline Stankosky (2005) & $\begin{array}{l}\text { El aprendizaje puede describirse como la adquisición de conocimiento o una habilidad a través del } \\
\text { estudio, la experiencia o la instrucción. El aprendizaje organizacional debe abordarse con enfoques } \\
\text { tales como el aumento de la comunicación interna, la promoción de equipos multifuncionales y la } \\
\text { creación de una comunidad de aprendizaje. El aprendizaje es una parte integral de la gestión del } \\
\text { conocimiento. }\end{array}$ \\
\hline Pradhan, Jena y Singh (2017) & $\begin{array}{l}\text { El aprendizaje organizacional ayuda a que la organización pueda generar nuevas estrategias para } \\
\text { incrementar su rendimiento por medio de las experiencias aprendidas. }\end{array}$ \\
\hline Weinzimmer y Esken (2017) & $\begin{array}{l}\text { El aprendizaje organizacional es importante para desarrollar una administración basada en el } \\
\text { cambio y la adquisición de nuevas prácticas para adaptarse al entorno. }\end{array}$ \\
\hline Liu (2018) & $\begin{array}{l}\text { Es un proceso fundamental para responder a los cambios en el entorno de la organización, con el } \\
\text { objetivo de mejorar la eficiencia de la transmisión del conocimiento y aprovechar las oportunidades } \\
\text { del mercado. }\end{array}$ \\
\hline Qi y Chau (2018) & $\begin{array}{l}\text { Para los autores este término se puede usar con el fin de medir el desempeño de las organizaciones y } \\
\text { hace referencia a la forma en la que la organización construye, almacena y organiza su conocimiento } \\
\text { y rutinas, a través de sus actividades y la cultura organizacional que esta tiene. }\end{array}$ \\
\hline
\end{tabular}

Fuente. Elaboración propia. 
Puede verse en las definiciones de la tabla 1 como el concepto de aprendizaje organizacional involucra, en sus inicios, la aplicación de conocimientos para el beneficio de la organización; posteriormente, se incluyen otros conceptos alrededor de este tales como la creación, la adquisición y la transferencia de conocimiento, lo que se traduce en cambios hacia los procesos organizacionales.

\subsection{Modelos de aprendizaje organizacional}

Para Nonaka y Takeuchi (1995), el conocimiento se puede dar de dos formas: tácito o explícito. El tácito es personal, difícil de manifestar, de expresar y de compartir con otros. El explícito es verbal, procesable fácilmente por varias personas y puede convertirse en documento. El modelo muestra que, de la interacción entre estas dos formas de aprendizaje, surgen las cuatro formas de conversión del conocimiento que ya se mencionaron: la socialización, la exteriorización, la combinación y la interiorización.

Figura 2. Modelo de aprendizaje de Nonaka y Takeuchi
En la socialización, el aprendizaje de tácito individual a tácito colectivo tiene la clave en la experiencia; la segunda es la exteriorización, de tácito a explícito, por la cual se convierte el conocimiento en metáforas, analogías, conceptos, hipótesis y modelos; la tercera forma de conversión es la combinación, de explícito a explícito, al sistematizar los conceptos y reconfigurar la información; por último, se encuentra la interiorización, en la que se pasa de explícito a tácito, es decir, se aprende haciendo. De cada forma surge un tipo de conocimiento: de la socialización el conocimiento armonizado; de la exteriorización, el conocimiento conceptual; de la combinación el conocimiento sistémico, y de la interiorización el conocimiento operacional.

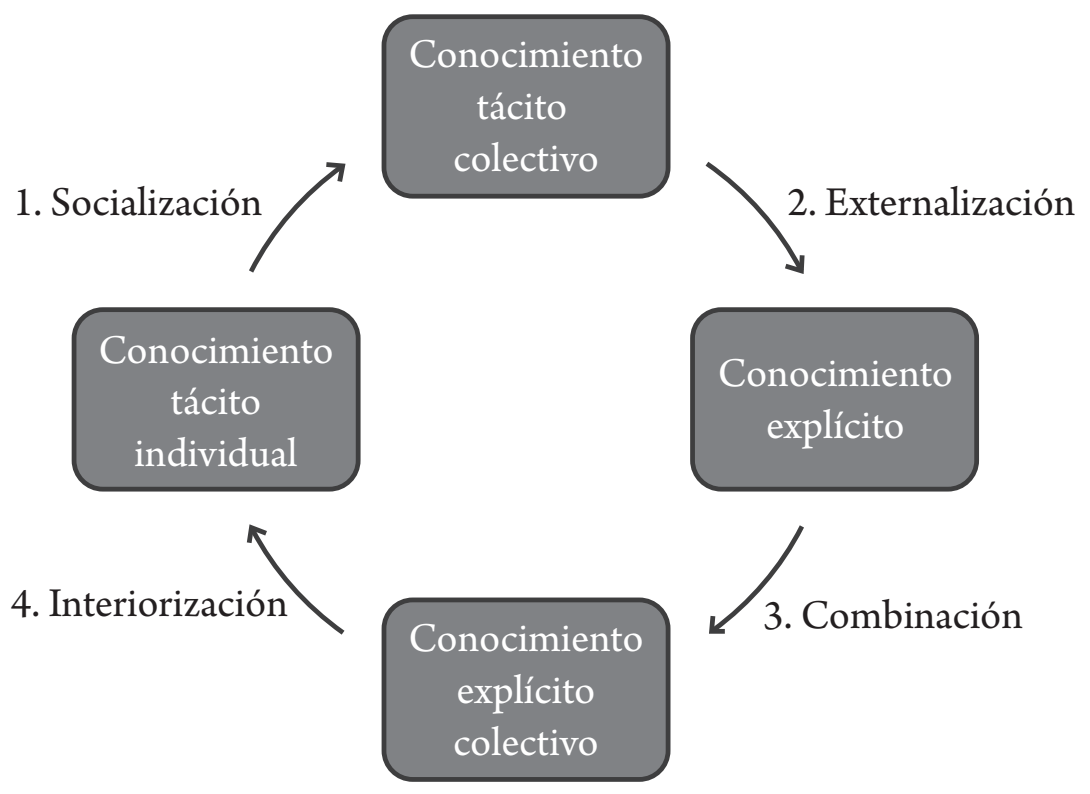

Fuente. Nonaka y Takeuchi, 1995. 
Otro modelo a destacar es el planteado por Kolb et al. (2001) sobre la «teoría de aprendizaje experiencial». Este se basa en la importancia del papel que juega la experiencia en el proceso de aprendizaje, cuando se construye conocimiento con reflexión y se da sentido a las experiencias. En este modelo se exploran los procesos cognitivos colaboradores a fin de abordar $y$ procesar las experiencias, de modo que se identifican y describen los diferentes estilos de aprendizaje (Gómez-Pawelek, 2003).

Para Kolb et al. (2001), el aprendizaje debe darse en cuatro etapas: 1) experiencia concreta, cuando hacemos algo; 2) observación reflexiva, cuando analizamos sobre lo hecho, reflexionamos sobre lo vivido y establecemos una conexión entre dichas etapas; 3) conceptualización abstracta, porque al reflexionar obtenemos conclusiones o generalizaciones más amplias con respecto a la situación vivida; 4) experimentación activa, esto es, cuando se desarrollan en la práctica las conclusiones obtenidas.

Los modelos mencionados permiten evidenciar la importancia de las experiencias de las personas en las organizaciones, por tanto, es indispensable para las empresas contar con dinámicas que puedan ser útiles para desarrollar el proceso de aprendizaje de la organización, es decir, la transmisión y sistematización de los conocimientos tácitos.

\subsection{Tecnologías de la información y las comunicaciones (TIC)}

Cobo-Romani (2009) en su investigación indica que muchas de las fuentes bibliográficas, tanto en español como las publicaciones de artículos en inglés, utilizan de manera indistinta los términos tecnologías de la información (TI), nuevas tecnologías de la información (NTI) o nuevas tecnologías de la información y la comunicación (NTIC); en inglés los términos más utilizados corresponden a information technologies (IT) e information comunication tecnologies (ICT). Fernández-Muñoz (2005) argumenta que las TIC pueden definirse, de acuerdo con un consenso colectivo, como innovaciones en diferentes campos tales como microelectrónica, computa- ción — hardware y software - o telecomunicaciones y optoelectrónica -microprocesadores, semiconductores, y fibra óptica- que permiten el procesamiento y la acumulación de enormes cantidades de información, además de una rápida distribución de la información a través de redes de comunicación.

La Ley 1341 del 30 de julio de 2009 del Congreso de la Republica de Colombia, en su artículo 6 define las tecnologías de la información y las comunicaciones (TIC) como «el conjunto de recursos, herramientas, equipos, programas informáticos, aplicaciones, redes y medios, que permiten la compilación, procesamiento, almacenamiento, transmisión de información como: voz, datos, texto, vídeo e imágenes».

De acuerdo con lo anterior, Aguilera-Castro y RiascosErazo (2009) consideran las TIC importantes aliadas con miras a los logros de la organización. Las autoras mencionan que, para la dirección estratégica, responsable de formular estrategias y planes de actuación empresarial eficientes y efectivos, es indispensable el recurso tecnológico, de manera que se facilite la comunicación entre la organización y el cliente —interno y externo- - Además, estas autoras desarrollaron una investigación en la que se evidencia que las TIC apoyan los procesos de gestión humana en diversas etapas: ingreso — reclutamiento, selección, contratación, inducción-, funciones —análisis de puestos, diseño de cargos-, compensación —retribución, plan de incentivos, beneficios-, adquisición de conocimientos —capacitación $y$ entretenimiento, formación y desarrollo- $y$ evaluación. (Riascos-Erazo y Aguilera-Castro, 2011).

En el estudio de Priyono (2016) se aborda el uso de las TIC en el contexto de las organizaciones basadas en proyectos y su intercambio de conocimiento; en específico, la aplicación de mensajería WhatsApp en los grupos de proyectos y su impacto en el rendimiento de estos, reflejado en una comunicación más efectiva, así como en el alcance de las metas y los objetivos de dichos equipos. Ante esto, Adamczewski (2016) también afirma que el desarrollo de las TIC en esta era se encuentra fuertemente relacionado con 
las herramientas de soporte al proceso gerencial en la organización, el cual necesita adaptar los métodos estratégicos de gestión a las nuevas condiciones empresariales de transformación digital, de manera que coincide con lo expuesto por Aguilera-Castro y Riascos-Erazo (2009).

Así mismo, Yunis, Tarhini y Kassar (2018) afirman que las tecnologías de la información y las telecomunicaciones han sido un elemento clave en el incremento del desempeño de las organizaciones, el crecimiento de la economía y el cambio social, lo que permite desarrollar nuevas estrategias innovadoras que se pueden utilizar dentro de las empresas y mejorar así aspectos tales como las relaciones entre los colaboradores y su rendimiento.

Chege, Wang y Suntu (2019) también consideran que las TIC han facilitado el desarrollo de las actividades en el interior de las organizaciones, de modo que se generan sitios de trabajo más interactivos. De acuerdo con el estudio que los autores realizaron, también se recomienda que el gobierno diseñe programas que promuevan el mejoramiento de la infraestructura tecnológica de las pymes, con el objetivo de contribuir al mejoramiento de las prácticas de estas compañías y su desempeño. Además de lo anterior, BernalJurado, Mozas-Moral, Medina-Viruel y FernándezUclés (2018) mencionan que un adecuado uso de las TIC en el desarrollo de estrategias relacionadas con e-commerce tiene un impacto positivo en el desempeño de la organización, en específico en términos de eficiencia económica.

\subsection{Relación entre aprendizaje organizacional, TIC y gestión del conocimiento}

Existe un cierto consenso en la literatura alrededor de que la gestión del conocimiento es un conjunto de prácticas interrelacionadas que generan valor a la organización y que están apoyadas por las tecnologías de la información y la comunicación, las cuales facilitan la adquisición, creación, distribución o diseminación, conversión y utilización de conocimiento dentro del contexto organizacional. Cardoso, Meireles y Peralta (2012), Cegarra-Navarro, Soto-Acosta y Wensley (2015), así como Palacios-Marqués, Soto-Acosta y Merigó (2015) también plantean que el papel central de las nuevas TIC radica en el apoyo que se brinda a los colaboradores para compartir y almacenar de manera electrónica el conocimiento.

Dentro de los espacios de aprendizaje de una organización se realizan los denominados «laboratorios de reclamos», en los cuales los participantes comprenden de forma sistémica el proceso y analizan lo relacionado con la solución de problemas, costos, etc., a través de plataformas que simulan la gestión de estos reclamos. De acuerdo con lo anterior, la utilización de las TIC es una potente herramienta para la gestión almacenamiento, organización, distribución, respuesta - de la información obtenida y su posterior utilización para beneficio de los procesos, como apoyo al aprendizaje organizacional y facilitando la gestión del conocimiento planteado por Stankosky (2005).

En la actualidad, diversos autores afirman que el aprendizaje organizacional, las TIC y la gestión del conocimiento tienen gran importancia en el desarrollo y el rendimiento organizacional. Argote y Hora (2017) argumentan que el aprendizaje organizacional tiene que incluir procesos de gestión de conocimiento a fin de que se pueda llevar a cabo, para lo cual las TIC ejercen un papel importante en el almacenamiento y la difusión de la información. Ahmad et al. (2017) concuerdan con lo anterior y añaden que dicho proceso tiene un impacto positivo en el rendimiento organizacional, a lo que Fang, Li y $\mathrm{Lu}$ (2016), Husain, Dayan y Di Benedetto (2016), así como Soto-Acosta y Cegarra-Navarro (2016) agregan que el uso adecuado de estos elementos puede ser uno de los pilares para la innovación dentro de las empresas, su competitividad y la adaptabilidad a los constantes cambios del entorno.

Desde el análisis de coocurrencia evidenciado en la figura 3 se logra reconocer una relación entre los conceptos TIC, aprendizaje organizacional y gestión del conocimiento - ICT, organizational learning and knowledge management - a lo largo del tiempo. Es importante destacar que, inicialmente —año 2009-, la comunidad académica abordó temas relacionados 
con tecnología de la información — color gris más claro-, entre el periodo 2009-2011 se profundizó el tema de gestión del conocimiento en gran medida, interesándose en aspectos relacionados con aprendizaje organizacional —color gris medio-, y, recientemente, la preocupación de académicos ha estado orientada hacia temáticas relacionadas con la transferencia de conocimiento - color gris oscuro-.
Por otra parte, se puede apreciar que los conceptos de aprendizaje organizacional ygestión del conocimiento se han venido relacionando desde el 2010 y existe una amplia investigacion sobre ellos —razón por la cual tienen el mayor tamaño-; aunque se evidencia que hay otros estudios que también relacionan estos elementos de investigación con otros tales como e-learning, rendimiento, pyme y ventaja competitiva.

Figura 3. Relación de los elementos a través del tiempo

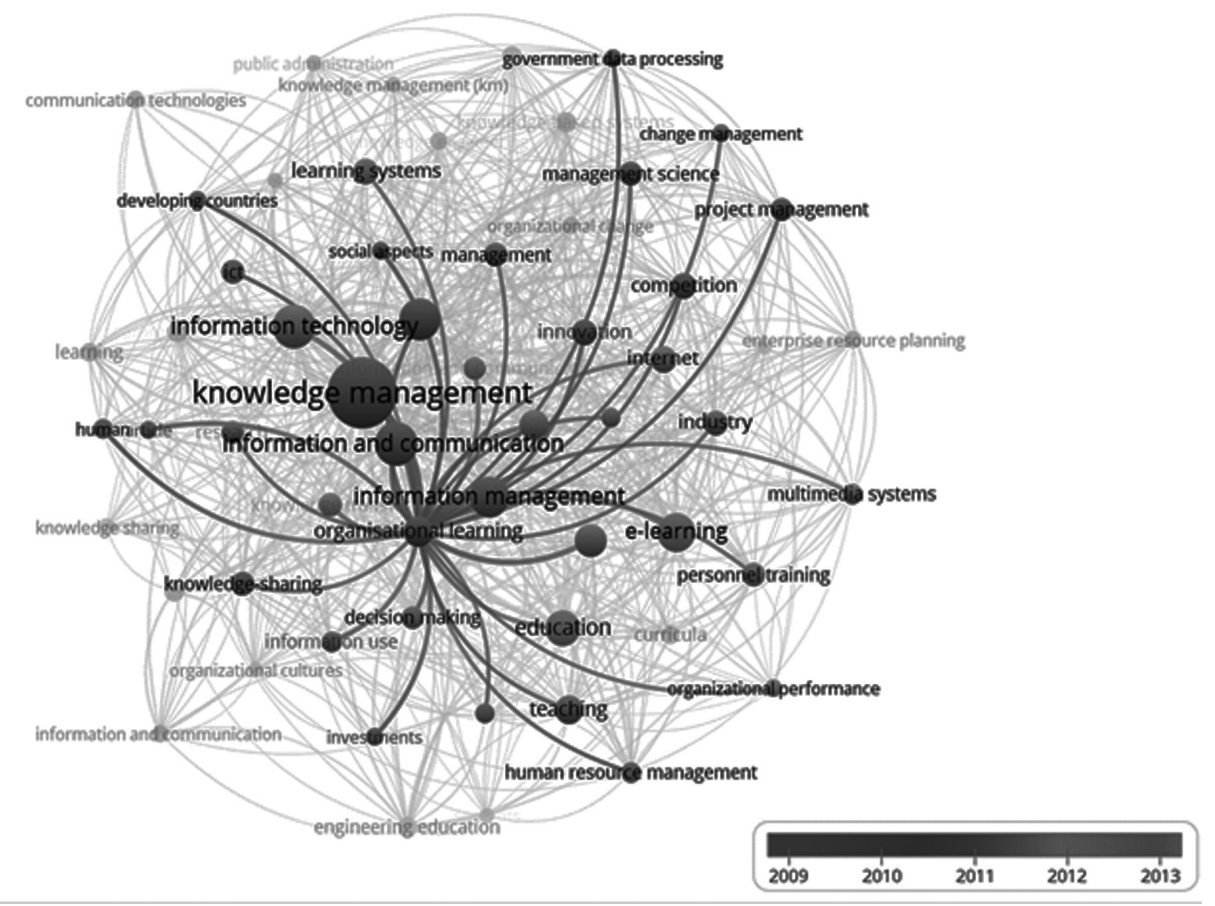

Fuente. Elaboración propia.

\section{Método}

$\mathrm{L}$ a investigación se realizó con un enfoque cuantitativo, es decir, el estudio realizó la aplicación de recolección de datos y el análisis estadístico con el fin de establecer patrones de comportamiento (Hernández-Sampieri, FernándezCollado y Baptista-Lucio, 2010). También responde a las características de un estudio descriptivo en el que se especifican propiedades y características de los procesos, objetos o cualquier otro fenómeno que se someta a un análisis (Hernández-Sampieri et al., 2010). La información recolectada se transformó en datos cuantitativos que permitieron aplicar 
herramientas estadísticas y facilitaron desarrollar correlaciones entre las prácticas de aprendizaje y las tecnologías desarrolladas a nivel de gestión de conocimiento en las pymes del Valle del Cauca, para luego proponer estrategias.

El estudio se realizó con una muestra de 117 pymes de 7903 registradas en Cámara de Comercio de Cali en el 2017, que respondió a un $95 \%$ de confiabilidad y un $9 \%$ de error, de acuerdo con la fórmula de tamaño de la muestra (véase la Figura 4). Para la recolección de la información se elaboró una encuesta que se caracterizó por tener preguntas cerradas y su escala de valoración fue la escala de Likert.

Figura 4. Fórmula de tamaño de la muestra

$$
\mathrm{n}=\frac{\mathrm{n}_{\mathrm{o}}}{1+\frac{\mathrm{n}_{\mathrm{o}}}{\mathrm{N}}} \text { donde: } \mathrm{n}_{\mathrm{o}}=p^{*}(1-p)^{*}\left\{\frac{\mathrm{Z}\left(1-\frac{\alpha}{2}\right)}{\mathrm{d}}\right\}^{2}
$$

Fuente. Elaboración propia.

\subsection{Variables del estudio}

A fin de obtener la información correspondiente a cada una de las variables se efectuaron preguntas que resultaron del referente teórico construido en el estudio. En la tabla 2 se esquematizan las preguntas utilizadas para validar cada una de las variables del estudio.

Tabla 2. Variables del estudio

\begin{tabular}{|c|c|c|}
\hline Variable & Descripción & Preguntas \\
\hline $\begin{array}{c}\text { Gestión del } \\
\text { conocimiento }\end{array}$ & $\begin{array}{l}\text { Conjunto de actividades } \\
\text { direccionadas a visionar, } \\
\text { diseñar, crear y liderar el } \\
\text { manejo del conocimiento } \\
\text { tácito y explícito en las } \\
\text { organizaciones (Von } \\
\text { Krogh, Ichijo y Nonaka, } \\
2001 \text { ). }\end{array}$ & $\begin{array}{l}\text { 9.1 La empresa diseña estrategias y políticas para los colaboradores con el fin de } \\
\text { desarrollar proyectos. } \\
\text { 9.2 La empresa motiva a sus colaboradores a trabajar en equipo en las distintas } \\
\text { actividades que realizan. } \\
\text { 9.3 Alienta la empresa a sus colaboradores a generar ideas y expresar en grupo sus } \\
\text { opiniones con total libertad. } \\
\text { 9.4 En la empresa se fomenta la reflexión, la crítica y el cambio para la mejora } \\
\text { continua y la resolución de problemas. } \\
\text { 9.5 Percibe que los cambios en la organización son positivos al gestionar el } \\
\text { conocimiento. } \\
\text { 9.6 Existen mecanismos que incentiven al personal de la organización para que } \\
\text { generen nuevas ideas de negocio. }\end{array}$ \\
\hline
\end{tabular}




\begin{tabular}{|c|c|c|}
\hline $\begin{array}{c}\text { Aprendizaje } \\
\text { organizacional }\end{array}$ & $\begin{array}{l}\text { Capacidad de una } \\
\text { organización de } \\
\text { transformar la } \\
\text { información que recibe } \\
\text { tanto del ambiente } \\
\text { externo como interno en } \\
\text { conocimiento; es decir, } \\
\text { la reconoce, clasifica, } \\
\text { almacena y analiza con el } \\
\text { objetivo de provocar un } \\
\text { resultado positivo. }\end{array}$ & $\begin{array}{l}\text { 5.4 La capacitación externa, como, por ejemplo, seminarios u otros, ¿ha mejorado la } \\
\text { experiencia de los colaboradores en el área de desempeño? } \\
\text { 5.5. ¿Las técnicas de benchmarking -recopilación de información con los } \\
\text { competidores- han permitido aprender de los éxitos y los fracasos de otras } \\
\text { empresas? } \\
6.1 \text { ¿El apoyo entre colaboradores y la colaboración mutua ha permitido la } \\
\text { transferencia de conocimiento? } \\
6.5 \text { ¿La empresa genera entrenamiento basado en nuevas metodologías o tecnologías } \\
\text { para mejorar el aprendizaje? } \\
7.2 \text { ¿Resulta fácil compartir con los compañeros de trabajo la experiencia y los } \\
\text { conocimientos adquiridos en la empresa? } \\
8.1 \text { ¿Los colaboradores se benefician del conocimiento de la empresa y de los } \\
\text { funcionarios más experimentados? } \\
13.13 \text { ¿Falta voluntad y confianza para compartir conocimiento? } \\
13.14 \text { ¿Falta de capacidad para transmitir y compartir conocimiento? } \\
13.15 \text { ¿Falta experiencia en el intercambio de conocimiento? } \\
13.16 \text { ¿Existe ausencia de cultura de intercambio de conocimiento? }\end{array}$ \\
\hline Tecnología & $\begin{array}{l}\text { Conjunto de teorías y de } \\
\text { técnicas que permiten } \\
\text { el aprovechamiento } \\
\text { práctico del } \\
\text { conocimiento científico }\end{array}$ & $\begin{array}{l}\text { 3.4 ¿Considera que la empresa ha crecido tecnológicamente en los últimos años? } \\
\text { 5.6. ¿Considera que las tecnologías de información -internet, intranet, redes } \\
\text { sociales, equipos móviles, etc.-le han permitido adquirir nuevos conocimientos? } \\
\text { 7.4 Los colaboradores, a través de la tecnología, ¿comparten regularmente el } \\
\text { conocimiento con sus compañeros y superiores? } \\
7.5 \text { ¿La empresa utiliza las tecnologías de la información como medio para transmitir } \\
\text { y compartir el conocimiento? } \\
11.1 \text { ¿Considera que el uso de las tecnologías de la información va en línea con la } \\
\text { estrategia de la empresa? } \\
11.2 \text { Las herramientas electrónicas y aplicaciones como e-mail, Skype y otros, } \\
\text { ¿generan eficiencia en la empresa? } \\
11.3 \text { ¿La empresa cuenta con un área formal de sistemas y con un personal } \\
\text { permanente para atender los requerimientos? } \\
11.4 \text { ¿La empresa cuenta con una plataforma tecnológica para compartir información } \\
\text { yapoyar la gestión en el conocimiento? } \\
11.5 \text { En el diseño de la estrategia, ¿la empresa considera la inversión tecnológica? } \\
13.5 \text { ¿Faltan herramientas de comunicación eficientes en el interior de la empresa? } \\
13.6 \text { ¿Faltan colaboradores cualificados en el manejo de la tecnología? } \\
13.7 \text { ¿La inversión es poca en recursos tecnológicos para la gestión del conocimiento? } \\
13.8 \text { ¿Los sistemas de información son obsoletos? }\end{array}$ \\
\hline
\end{tabular}

Fuente. Elaboración propia.

$\mathrm{Al}$ considerar la importancia que tiene la validez de contenido del instrumento para la generación de aportes investigativos (Cronbach y Meehl, 1955), el estudio aplicó la técnica de juicio de expertos en la temática de gestión del conocimiento (Barrazas, 2007; Escobar-Pérez y Cuervo-Martínez, 2008). Su aplicación consistió en contactar a cinco expertos, posteriormente enviar el instrumento por correo 
electrónico y, finalmente, recibir los aportes y consideraciones, lo que permitió generar una versión final del instrumento. Entre las observaciones recibidas se destaca: la adición de preguntas $-9,4$;
13,16 y $13,5-$ y el concepto de la pertinencia del cuestionario para establecer la relación aprendizaje organizacional, TIC y gestión del conocimiento.

\section{Resultados y discusión}

\subsection{Confiabilidad y validez de instrumentos}

T a primera fase del análisis de los resultados obtenidos fue la realización de la prueba de fiabilidad del _uestionario a través de la prueba de alfa de Cronbach (George y Mallery, 2003), de la que se obtuvieron los resultados que se presentan en la tabla 3.

Tabla 3. Alfa de Cronbach

\begin{tabular}{|c|c|c|c|c|c|}
\hline \multicolumn{2}{|c|}{$\begin{array}{c}\text { Cooeficiente de fabilidad } \\
\text { tecnología }\end{array}$} & \multicolumn{2}{c|}{$\begin{array}{c}\text { Coeficiente de fiabilidad } \\
\text { aprendizaje }\end{array}$} & \multicolumn{2}{c|}{$\begin{array}{c}\text { Coeficiente de fiabilidad gestión dell } \\
\text { conocimiento }\end{array}$} \\
\hline Alfa de Cronbach & $\mathrm{N}^{\circ}$ de preguntas & Alfa de Cronbach & $\mathrm{N}^{\circ}$ de preguntas & Alfa de Cronbach & $\mathrm{N}^{\circ}$ de preguntas \\
\hline 0.80 & 13 & 0.75 & 11 & 0.91 & 6 \\
\hline
\end{tabular}

Fuente. Elaboración propia.

De acuerdo con George y Mallery (2003), el nivel de fiabilidad del cuestionario, de acuerdo con el estadístico alfa de Cronbach, es bueno para la variable tecnología, aceptable para la variable aprendizaje y excelente para gestión del conocimiento, considerando que estos niveles están por encima de 0,8, 0,7 y 0,9, respectivamente.

\subsection{Validez de constructo}

Para la validación del constructo se realizó un análisis factorial por el que se obtuvo como primer resultado, ofrecido por la prueba de KMO (Kaiser-Meyer-Olkin) y Bartlett el valor de 0,894, lo cual significa que es viable utilizar el método de análisis factorial con los datos recolectados en el estudio (véase la Tabla 4).

Tabla 4. Prueba de KMO y Bartletta

\begin{tabular}{|l|c|c|}
\hline \multirow{3}{*}{ Medida Kaiser-Meyer-Olkin de adecuación de muestreo } & 0,894 \\
\cline { 2 - 3 } Prueba de esfericidad de Bartlett & Aprox. Chi-cuadrado & 2693,747 \\
\cline { 2 - 3 } & gl & 435 \\
\cline { 2 - 3 } & Sig. &, 000 \\
\hline \multicolumn{2}{|c}{ a. Se basa en correlaciones } \\
\hline
\end{tabular}

Fuente. Elaboración propia.

Posteriormente, se presenta la tabla 5 relacionada con la matriz de varianza que permite evidenciar un porcentaje acumulado del 63 \% para la explicación de los componentes analizados en el estudio; esto quiere decir que, aunque superior al $50 \%$, permite validar el constructo que subyace la variable de interés relacionada con la gestión del conocimiento a partir del estudio del aprendizaje organizacional y las TIC. 
Tabla 5. Varianza total explicada

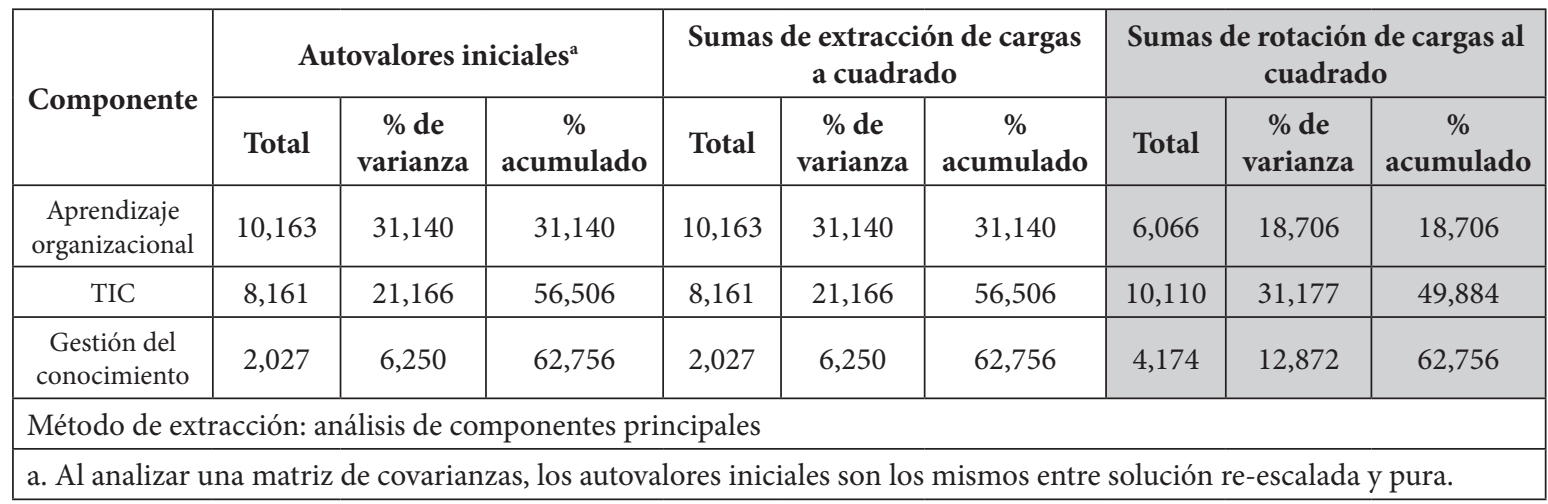

Fuente. Elaboración propia.

Por otra parte, se realizó un análisis de la relación de las variables a través del estadístico Test V de Cramer, cuyos resultados se ilustran en la figura 5. En esta se puede evidenciar mayor relación entre los resultados de la variable aprendizaje y la gestión del conocimiento, en la cual se ilustran nueve relaciones con un valor mayor a 0,5 , a diferencia de la relación entre TIC y gestión del conocimiento, en la cual solo se evidencian tres relaciones mayores a 0,5 ; estos valores se identifican subrayados en la figura 5 .

Figura 5. Test V de Cramer por pares de variables para los pilares tecnología, aprendizaje y gestión del conocimiento

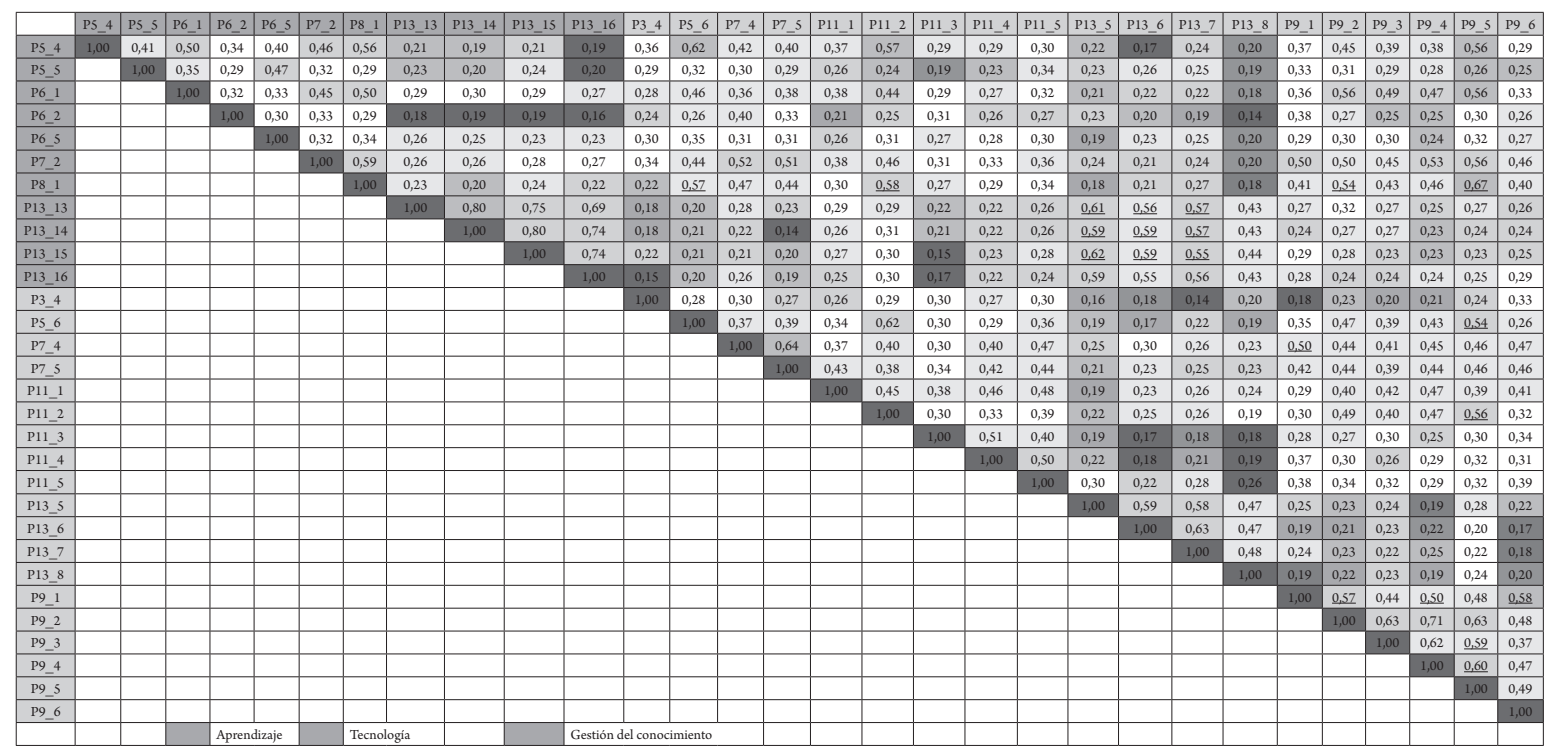

Fuente. Elaboración propia.

Se destaca que, en las pymes consultadas, la relación entre la percepción positiva de la organización frente a gestionar el conocimiento y el beneficio que obtienen los colaboradores del conocimiento de la empresa y de los funcionarios más experimentados es notoria - 0,66 - Es posible, entonces, afirmar que esta correlación puede presentarse en la organización de forma cíclica, debido a que el proceso de beneficiarse del conocimiento existente en la empresa, así como de los colaboradores más experimentados, redunda en formas más eficientes y eficaces de realizar el trabajo. Esto, a su vez, proporciona nuevo conocimiento organizacional, en el cual se basarán otros colaboradores. De acuerdo con Senge 
(1999), este aprendizaje, edificado a partir de las experiencias organizacionales, constituye la memoria de la organización, la cual debe ser documentada y resguardada de forma adecuada para consulta y uso posterior de los colaboradores.

Así mismo, la percepción de las pymes frente a la gestión del conocimiento se relaciona de forma notoria - 0,57- con el apoyo entre colaboradores, $y$ la colaboración mutua le ha permitido la transferencia de conocimiento. Esta correlación se evidencia en la dinámica del proceso de los sistemas o programas de gestión del conocimiento en las organizaciones que contribuyen a gestionar de manera adecuada los cambios que en ellas surgen. De igual forma, es importante no perder de vista que la transferencia de conocimiento, el apoyo entre colaboradores y la gestión de los cambios organizacionales están fuertemente influidos por la cultura de la organización. De acuerdo con Jennex y Olfman (2004), esta debe ser soporte, además, para los procesos de aprendizaje y uso del conocimiento.

En cuanto a la relación entre TIC y gestión del conocimiento se destaca la relación de 0,56 entre la percepción que las pymes tienen de los cambios que puede generar el gestionar el conocimiento para la organización y la utilización de herramientas electrónicas y aplicaciones como, por ejemplo, el e-mail, Skype u otros, lo que genera de manera especial eficiencia en sus procesos y la adquisición de nuevos conocimientos.

Esta correlación se enfoca en dos conceptos: el primero de ellos referente a la eficiencia de los procesos a partir de la utilización de las TIC como herramienta de mediación en la comunicación, y la otra, en términos de la adquisición de conocimientos también mediada por las TIC. Esto se muestra acorde al consenso que existe en los estudios de varios autores que consideran las TIC un soporte a la gestión del conocimiento con miras a compartir, almacenar, distribuir, adquirir, convertir y utilizar este conocimiento (Cardoso et al., 2012; Cegarra-Navarro et al., 2015; Palacios-Marqués, Soto-Acosta y Merigó, 2015).
Por otra parte, los resultados del Test de Cramer permiten resaltar una correlación de 0,5 en los siguientes aspectos: la empresa diseña estrategias y políticas para los colaboradores con el fin de desarrollar proyectos - gestión del conocimiento- $-y$ los colaboradores a través de la tecnología comparten regularmente el conocimiento con sus compañeros y superiores - aspecto tecnológico-. En este contexto cabe anotar que las estrategias y políticas presentadas por las organizaciones para el desarrollo de proyectos brindan a los colaboradores espacios para el trabajo en equipo, la generación, transmisión e incluso el almacenamiento de conocimiento organizacional. Es posible, en este sentido, establecer un paralelo con los «micromundos» planteados por Senge (1999), en los cuales las organizaciones inteligentes logran poner a disposición de sus colaboradores espacios de aprendizaje en los que se evalúan, desarrollan e implementan nuevas estrategias, se analizan oportunidades y se desarrollan proyectos.

Finalmente, se evidencia a través de estos resultados que las pymes objeto de estudio han avanzado en dinámicas de aprendizaje organizacional y de estrategias tecnológicas para apoyar el proceso de gestionar el conocimiento. Sin embargo, es necesario aportar algunos elementos que permitan subsanar dificultades identificadas en las pymes estudiadas, pues, considerando lo mencionado por Choi (2004), el entrenamiento y la capacitación de los colaboradores constituye uno de los aspectos más importantes para la implementación y el éxito de los programas de gestión del conocimiento, así como la infraestructura tecnológica -integración aprendizaje organizacional y tecnología-.

\subsection{Estrategias para fortalecer la relación aprendizaje organizacional y TIC como apoyo a la gestión del conocimiento}

De acuerdo con los resultados del estudio, se evidencia que las pymes del Valle del Cauca requieren una guía o un direccionamiento estratégico para implementar procesos de gestión del conocimiento en las organizaciones. Actualmente, las pymes implementan estrategias generales que de forma directa apoyan la gestión del conocimiento. 
Es importante considerar que, a nivel de política pública, en Colombia el Ministerio de las TIC tiene como propósito «consolidar la adopción de las TIC en las mipymes del país, como un medio para el aumento de su productividad y competitividad» (MinTIC, s. f.); esto podría ayudar a las pymes a fortalecer sus aspectos tecnológicos, lo que permitirá apoyar los procesos de gestión del conocimiento de la organización.

En la tabla 6 se proponen estrategias para fortalecer las prácticas de gestión de conocimiento en el contexto de las pymes del Valle del Cauca, de acuerdo con los resultados obtenidos y las debilidades detectadas que permitan alcanzar una competitividad estratégica, lo cual es la fórmula que genera valor y se aplica con éxito (Hitt, Duane-Ireland y Hoskisson, 2004).

La metodología bajo la cual se proponen algunas estrategias para la gestión del conocimiento consiste, inicialmente, en el planteamiento de objetivos estratégicos y, posteriormente, de las acciones a seguir para su cumplimiento.

Tabla 6. Objetivos estratégicos para las prácticas de aprendizaje organizacional y tecnologías de la información y las comunicaciones

\begin{tabular}{|c|c|}
\hline Objetivo estratégico & Acciones \\
\hline $\begin{array}{l}\text { Mejorar los mecanismos y las prácticas } \\
\text { para apoyar la transferencia de conoci- } \\
\text { miento desde los colaboradores más ex- } \\
\text { perimentados, los cuales representan una } \\
\text { valiosa fuente de conocimiento organiza- } \\
\text { cional. }\end{array}$ & $\begin{array}{l}\text { - Programa de padrinazgo o mentoría: el personal de mayor experiencia adopta uno } \\
\text { o varios colaboradores, guiándolos y orientándolos por un periodo determinado en } \\
\text { sus labores cotidianas. } \\
\text { - Capacitaciones: motivar a los colaboradores experimentados para que compartan } \\
\text { los conocimientos adquiridos a través de capacitaciones, jornadas de entrenamiento } \\
\text { y foros, entre otras actividades formativas. Estas actividades se logran al brindar } \\
\text { incentivos emocionales y monetarios por su colaboración. } \\
\text { Documentación de la experiencia: esta práctica puede desarrollarse mediante } \\
\text { la implementación de videos cortos, fáciles de almacenar y distribuir; formatos } \\
\text { institucionales creados para la consignación escrita de dichas experiencias; } \\
\text { implementación de repositorios digitales o bancos de documentación, en los que } \\
\text { se puedan almacenar «lecciones aprendidas», etc. }\end{array}$ \\
\hline $\begin{array}{l}\text { Reforzar la cultura organizacional con } \\
\text { actividades que fomenten el intercambio } \\
\text { de conocimiento, fortaleciendo su gestión } \\
\text { en el interior de la empresa. }\end{array}$ & $\begin{array}{l}\text { - Establecer comités de trabajo con una frecuencia de reunión determinada. Los } \\
\text { comités son valiosos espacios de transferencia e intercambio de conocimiento, } \\
\text { proposición de ideas y solución a problemas, retroalimentación a los colaboradores } \\
\text { miembros del equipo acerca de la labor desempeñada y las tareas asignadas; estos } \\
\text { promueven, además, la cultura colaborativa dentro de la organización. } \\
\text { - Promover foros por procesos o equipos en los que se generen propuestas para la } \\
\text { solución a problemas que impactan la productividad o eficiencia de esos procesos. } \\
\text { Los foros deben ser documentados y otorgar visibilidad a los proponentes de ideas } \\
\text { y soluciones creativas e innovadoras. Los equipos pueden estar conformados con } \\
\text { miembros de diferentes procesos de la organización, en busca de aportes y puntos } \\
\text { de vista diferentes que enriquezcan el proceso. } \\
\text { Organizar actividades en las que se recompense el compartir conocimiento: por } \\
\text { ejemplo, premiar los equipos o colaboradores que logren consignar la mayor } \\
\text { cantidad de lecciones aprendidas; que tengan al final de un periodo determinado } \\
\text { el mayor número de acciones o planes de mejora para su proceso; que hayan } \\
\text { consignado en un determinado repositorio la mayor cantidad de capacitaciones - } \\
\text { en formato electrónico-, entre otras. }\end{array}$ \\
\hline
\end{tabular}




\begin{tabular}{|c|c|}
\hline $\begin{array}{l}\text { Establecer una metodología para la gestión } \\
\text { del conocimiento en la que se articulen } \\
\text { conceptos, herramientas y procedimientos } \\
\text { que brinden claridad respecto a las } \\
\text { políticas de la organización. }\end{array}$ & $\begin{array}{l}\text { - Integrar un equipo de trabajo que se encargue de la implementación de un sistema } \\
\text { de gestión del conocimiento adaptado a la organización, de manera que se inicie } \\
\text { con el diagnóstico de las prácticas que la empresa posee, así como por determinar } \\
\text { cuáles pueden ser adoptadas. } \\
\text { - Desarrollar programas de sensibilización para todo el personal de la organización } \\
\text { en los que se apropie el modelo de gestión del conocimiento de la organización. }\end{array}$ \\
\hline $\begin{array}{l}\text { Fortalecer el entorno tecnológico de la } \\
\text { organización con enfoque en los procesos } \\
\text { de capacitación y aprendizaje de los } \\
\text { colaboradores tanto para el desarrollo } \\
\text { como para el refuerzo de competencias } \\
\text { que impacten su crecimiento y adecuado } \\
\text { desempeño. }\end{array}$ & $\begin{array}{l}\text { - Disponer de equipos de cómputo en los cuales los colaboradores puedan ingresar a } \\
\text { formación virtual en distintas plataformas externas, disponiendo de las aplicaciones } \\
\text { necesarias para el desarrollo exitoso de dicho proceso. } \\
\text { - Generar campañas que promuevan el uso de herramientas tales como el correo } \\
\text { electrónico, a través del cual se pueden generar procesos de formación y aprendizaje. } \\
\text { Examinar las herramientas tecnológicas que existen en la organización y pueden } \\
\text { promover el aprendizaje y la gestión del conocimiento. Algunos ejemplos son: } \\
\text { internet, para conectividad con portales externos de formación y compartir } \\
\text { capacitaciones, entre otros; Skype, para conferencias web, capacitaciones, foros, } \\
\text { comités de equipos, etc. }\end{array}$ \\
\hline $\begin{array}{l}\text { Implementar un área administrativa } \\
\text { para la gestión de TIC que contribuya al } \\
\text { fortalecimiento de los procesos operativos } \\
\text { tales como soporte y mantenimiento; a la } \\
\text { implementación de nuevas herramientas } \\
\text { y, en general, a la gestión administrativa } \\
\text { de planear, ejecutar, controlar y mejorar } \\
\text { continuamente. }\end{array}$ & $\begin{array}{l}\text { - Determinar el perfil idóneo para quien será responsable de la gestión de TIC. } \\
\text { - Desarrollar planes de trabajo a partir de establecer equipos responsables de su } \\
\text { implementación y ejecución. } \\
\text { - Determinar la frecuencia yla forma para realizar el seguimiento a la implementación } \\
\text { de TIC por parte del líder de área y del equipo de trabajo conformado. }\end{array}$ \\
\hline $\begin{array}{l}\text { Examinar la viabilidad para la } \\
\text { implementación de plataformas específicas } \\
\text { de gestión del conocimiento, las cuales } \\
\text { se especializan en procesos tales como } \\
\text { la transmisión, el almacenamiento y la } \\
\text { disponibilidad de la información, además } \\
\text { de apoyar los procesos de capacitación y } \\
\text { aprendizaje en la organización. }\end{array}$ & $\begin{array}{l}\text { - Realizar un análisis de diferentes plataformas que faciliten gestionar el conocimiento } \\
\text { de la organización. } \\
\text { Implementar plataformas de formación virtual de código abierto -como, por } \\
\text { ejemplo, Moodle, Dokeos, Claroline, Sakai—, las cuales generan un menor costo } \\
\text { de inversión y permiten el desarrollo de programas de formación a la medida de las } \\
\text { necesidades de la organización. }\end{array}$ \\
\hline $\begin{array}{l}\text { Capacitar al personal en aspectos puntuales } \\
\text { relacionados con la gestión de TIC que } \\
\text { faciliten procesos de implementación de } \\
\text { nuevas herramientas tecnológicas y la } \\
\text { gestión del conocimiento. }\end{array}$ & $\begin{array}{l}\text { Determinar la malla curricular de aprendizaje en la que se establezcan los temas que } \\
\text { deben aprender los colaboradores de la organización. } \\
\text { Establecer jornadas de capacitación teórico-prácticas que incentiven el aprendizaje } \\
\text { del personal vinculado. }\end{array}$ \\
\hline
\end{tabular}

Fuente. Elaboración propia.

\section{Conclusiones}

A través de la investigación realizada a las pymes del Valle del Cauca se logró identificar distintas prácticas que, desde los aspectos del aprendizaje organizacional y las tecnologías de la información y comunicación apoyan el proceso de gestión del conocimiento. Entre ellas cabe destacar la capacitación tanto interna como externa a los colaboradores y la transferencia de conocimiento entre colaboradores, así como el aprovechamiento por parte de los colaboradores del conocimiento que ha adquirido la pyme a través del tiempo para su desempeño en el cargo. 
El análisis de correlación permite concluir que se identifica en las pymes un mayor número de actividades de aprendizaje organizacional asociadas a la gestión del conocimiento, a diferencia de una minoría de actividades de TIC que se relacionan con actividades propias de la gestión del conocimiento. Esto corrobora lo mencionado por diversos investigadores, quienes señalan que las TIC no son una prioridad para las pymes.

$\mathrm{Si}$ se tienen en cuenta los resultados de esta investigación, se puede evidenciar que las pymes carecen de una metodología clara para el desarrollo de la gestión del conocimiento y los beneficios que con ella se generan. Por otra parte, también se hace evidente que las pymes del Valle del Cauca se encuentran construyendo sus áreas de gestión del conocimiento, en gran parte porque comenzaron la estructuración de políticas de tecnologías de la información que favorecen el crecimiento y el desarrollo organizacional, a partir de la evolución de la tecnología que, a su vez, permite la evolución de las formas de aprendizaje. Dentro de esta evolución se hace necesario que la organización lleve un registro que le permita establecer la frecuencia de actividades tales como capacitaciones, los temas abordados en ellas y la manera en que los participantes las asumen, a fin de que sea más fácil establecer la trayectoria y el impacto de los programas de capacitación.

Se sugiere contar con nuevas formas de crear y compartir conocimiento, como pueden ser los programas de padrinazgo o de mentores que permiten la transferencia de quienes tienen mayor experiencia hacia aquellos que apenas la están alcanzando. Además, como parte de un proceso idóneo, es recomendable la contratación de una consultoría externa que defina, gracias a su experticia, cuál es el sistema de gestión de conocimiento adecuado para la organización y las herramientas oportunas dirigidas a promover el aprendizaje y la gestión del conocimiento.

Los principales aportes del articulo están centrados en dos aspectos: el primero, evidencia claramente el avance de las pymes en prácticas de aprendizaje organizacional y las TIC como apoyo a la ges- tión del conocimiento, en el que se muestra cómo las pymes se encuentran en un nivel inicial en el proceso de gestionar su conocimiento. El segundo, las estrategias que se desprenden de los hallazgos encontrados en el estudio, en el que buscan, principalmente, potencializar la transferencia del conocimiento mediada por TIC a través de actividades de aprendizaje organizacional sistematizadas.

Se hace importante gestionar el conocimiento apoyado en la tecnología, pues esto permite ubicar la organización más cerca de la actualidad empresarial, el uso de herramientas tales como el correo electrónico, los foros o las redes sociales, entre otras, que facilitan la transferencia del conocimiento, dado que son un mecanismo efectivo y accesible a todos los colaboradores de manera corporativa. La tecnología y los planes de trabajo definidos se convierten en aliados infalibles para potenciar el aprendizaje organizacional y, por ende, la gestión del conocimiento que permitirá a las pymes consolidarse como organizaciones competitivas e innovadoras.

\section{Referencias}

Adamczewski, P. (2016). ICT solutions in intelligent organizations as challenges in a knowledge economy. Management, 20(2), 198-209.DOI: https://doi.org/10.1515/ manment-2015-0060

Aguilera-Castro, A.; Riascos Erazo, S. (2009). Direccionamiento estratégico apoyado en las TIC. Estudios Gerenciales, 25(111) 127-143. DOI: https://doi.org/10.1016/S01235923(09)70074-9

Ahmad, N.; Lodhi, M. S.; Zaman, K.; Naseem, I. (2017). Knowledge management: a gateway for organizational performance. Journal of the Knowledge Economy, 8(3), 859876. DOI: https://doi.org/10.1007/s13132-015-0282-3

Alavi, M.; Leidner, D. (2003). Sistemas de gestión del conocimiento: cuestiones, retos y beneficios. En S. Barnes (Ed.) Sistemas de gestión del conocimiento. Teoría y práctica. (17-40). España: Thompson Editores.

Argote, L.; Hora, M. (2017). Organizational learning and management of technology. Production and Operations Management,26(4),579-590.DOI: https://doi.org/10.1111/ poms. 12667 
Barrazas A. (2007). La consulta a expertos como estrategia para la recolección de evidencias de validez basadas en contenido. Investigación Educativa Duranguense, (7), 5-13.

Berawi, M. A. (2004). Quality revolution: Leading the innovation and competitive advantages. International Journal of Quality and Reliability Management, 21(4), 425-438. https://doi. org/10.1108/02656710410530118

Bernal-Jurado, E.; Mozas-Moral, A..; Medina-Viruel, M. J..; Fernández-Uclés, D. (2018). Evaluation of corporate websites and their influence on the performance of olive oil companies. Sustainability 10(4), 1274. DOI: https://doi.org/10.3390/ su10041274

Bibi, S.; Khan, A.; Qian, H.; Garavelli, A. C.; Natalicchio, A.; Capolupo, P. (2020). Innovative climate, a determinant of competitiveness and business performance in Chinese law firms: the role of firm size and age. Sustainability, 12(12), 4948. DOI: https://doi.org/10.3390/su12124948

Bilan, Y.; Hussain, H. I.; Haseeb, M.; Kot, S. (2020). Sustainability and economic performance: role of organizational learning and innovation. Engineering Economics, 31(1), 93-103. DOI: https://doi.org/10.5755/j01.ee.31.1.24045

Cámara de Comercio de Cali. (20 de agosto de 2019). Ritmo Empresarial I Cámara de Comercio de Cali. Recuperado de https://bit.ly/3qR0MzN

Cardoso, L.; Meireles, A.; Peralta, C. F. (2012). Knowledge management and its critical factors in social economy organizations. Journal of Knowledge Management, 16(2), 267284. DOI: https://doi.org/10.1108/13673271211218861

Cegarra-Navarro, J. G.; Soto-Acosta, P.; Wensley, A. (2015). Structured knowledge processes and firm performance: the role of organizational agility. Journal of Business Research, 69(5), 1544-1549. DOI: https://doi.org/10.1016/j. jbusres.2015.10.014

Chege, S. M., Wang, D.; Suntu, S. L. (2019). Impact of information technology innovation on firm performance in Kenya. Information Technology for Development, 26(2), 316-345. DOI: https://doi.org/10.1080/02681102.2019.1573717

Choi, Y. (2004). An empirical study of factors affecting successful implementation of knowledge management (Tesis de doctorado). Universidad de Nebraska, EE.UU. Recuperado de https://bit. ly/38HWuVa

Cobo-Romani, J. C. (2009). El concepto de tecnologías de la información. Benchmarking sobre las definiciones de las TIC en la sociedad del conocimiento. Zer: Revista de Estudios de Comunicación, 14(27), 295-318.

Cronbach, L. J.; Meehl, P. E. (1955). Construct validity in psychological tests. Psychological Bulletin, 52(4), 281-302. DOI: https://doi.org/10.1037/h0040957

Dalkir, K.; Liebowitz, J. (2011). Knowledge management in theory and practice. Massachusetts: MIT press.
Davenport, T. H.; Prusak, L. (2001). Conocimiento en acción: cómo las organizaciones manejan lo que saben. Argentina: Prentice Hall.

Drucker, P. F. (1999). Los desafíos de la gerencia para el siglo XXI. Editorial Norma.

Escobar-Pérez, J.; Cuervo-Martínez, Á. (2008). Validez de contenido y juicio de expertos: una aproximación a su utilización. Avances en Medición, 6(1), 27-36.

Escudero-Yerena, M. T. (1998). Técnicas de comunicación en la enseñanza. México: Trillas.

Etzioni, A. (1964). Organizaciones modernas. Madrid: Editorial Utea.

Fang, E. A.; Li, X.; Lu, J. (2016). Effects of organizational learning on process technology and operations performance in mass customizers. International Journal of Production Economics, (174), 68-75. DOI: https://doi.org/https://doi. org/10.1016/j.ijpe.2016.01.019

Fernández-Muñoz, R. (2005). Marco conceptual de las nuevas tecnologías aplicadas a la educación. España: La Mancha.

Ferraris, A.; Santoro, G.; Dezi, L.(2017). How MNC's subsidiaries may improve their innovative performance? The role of external sources and knowledge management capabilities. Journal of Knowledge Management, 21(3), 540-552. DOI: https://doi.org/10.1108/JKM-09-2016-0411

Fiol, M. C.; Lyles, M. (1985). Organizational learning. Academy of Management Review, 10(4), 803-813. DOI: https://doi. org/10.2307/258048

George, D.; Mallery, P. (2003). SPSS for Windows step by step: a simple guide and reference. Boston: Allyn \& Bacon.

Gómez-Pawelek, J. (2003). El aprendizaje experiencial. Buenos Aires, Argentina. Recuperado de https://bit.ly/3lq113U

Hernández-Sampieri, R., Fernánde-Collado, C.; Baptista-Lucio, P. (2010). Metodología de la investigación. México: Mc Graw Hill

Hindasah, L.; Nuryakin, N. (2020). The relationship between organizational capability, organizational learning and financial performance. Journal of Asian Finance, Economics and Business, 7(8), 625-633. DOI: https://doi.org/10.13106/jafeb.2020. vol7.no8.625

Hitt, M.; Duane-Ireland, R.; Hoskisson, R. E. (2004). Administración estratégica. Competitividad y conceptos de globalización (5 $5^{\mathrm{a}}$ ed.). México D. F.: International Thomson Editores.

Husain, Z.; Dayan, M.; Di Benedetto, C. A. (2016). The impact of networking on competitiveness via organizational learning, employee innovativeness, and innovation process: A mediation model. Journal of Engineering and Technology Management, (40), 15-28. DOI: https://doi.org/10.1016/j. jengtecman.2016.03.001 
Jennex, M.; Olfman, L. (2004). Assessing knowledge management success/effectiveness models. 37th Annual Hawaii International Conference on System Sciences. IEEE, Big Island, EE.UU., 5-8 de enero. DOI: https://doi.org/10.1109/ HICSS.2004.1265571

Kluge, A.; Schilling, J. (2000). Organizational learning and the learning organization: an overview of current theory and empirical results. Zeitschrift für Arbeits- und Organisationspsychologie A\&O, 44(4), 179-191.DOI: https:// doi.org/10.1026//0932-4089.44.4.179

Kolb, D. A.; Boyatzis, R. E.; Mainemelis, C. (2001). Experiential learning theory: previous research and new directions. En R. Sternberg; L-F. Zhang (Eds.) Perspectives on thinking, learning, and cognitive styles. (227-247). Nueva York: Routledge. DOI: https://doi.org/10.4324/9781410605986

Koulopoulos, T. M.; Frappaolo, C. (2001). Smart. Lo fundamental y lo más efectivo acerca de la Gerencia del Conocimiento. Bogotá: McGraw-Hill.

Krogh, G. von; Ichijo, K.; Nonaka, I. (2001). Facilitar la creación de conocimiento: cómo desentrañar el misterio del conocimiento tácito y liberar el poder de la innovación. México: Oxford University Press.

Ley 1341 de 2009. (29 de julio de 2011). Por la cual se definen Principios y conceptos sobre la sociedad de la información y la organización de las Tecnologías de la Información y las Comunicaciones - TIC_, se crea la Agencia Nacional del Espectro y se dictan otras disposiciones. Diario Oficial, núm 47.426. Congreso de Colombia.

Liu, C.-H. S. (2018). Examining social capital, organizational learning and knowledge transfer in cultural and creative industries of practice. Tourism Management, (64), 258270. DOI: https://doi.org/https://doi.org/10.1016/j. tourman.2017.09.001

Ministerio de Tecnologías de la Información y las Comunicaciones (MinTIC). (s. f.). Líneas estratégicas. Recuperado de https://mintic.gov.co/portal/vivedigital/612/w3-propertyvalue-19437.html

Muñoz, B.; Riverola, J. (2003). Del buen hacer y mejor pensar: mejora permanente y gestión del conocimiento. Madrid: McGraw-Hill.

Nie, K.; Ma, T.; Nakamori, Y. (2009). An approach to aid understanding emerging research fields - the case of knowledge management. Systems Research and Behavioral Science: The Official Journal of the International Federation for Systems Research, 26(6), 629-643. DOI: https://doi.org/10.1002/sres.926

Nonaka, I.; Takeuchi, H. (1995). The knowledge-creating company: how Japanese companies create the dynamics of innovation. Nueva York: Oxford University Press. DOI: https://doi. org/10.1016/0024-6301(96)81509-3
Palacios-Marqués, D.; Soto-Acosta, P.; Merigó, J. (2015). Analyzing the effects of technological, organizational and competition factors on Web knowledge exchange in SMEs. Telematics and Informatics, 32(1), 23-32. DOI: https://doi. org/10.1016/j.tele.2014.08.003

Papa, A.; Dezi, L.; Gregori, G. L.; Mueller, J.; Miglietta, N. (2018). Improving innovation performance through knowledge acquisition: the moderating role of employee retention and human resource management practices. Journal of Knowledge Management, 24(1). DOI: https://doi.org/10.1108/JKM-092017-0391

Pérez-Uribe, R. I.; Ramírez, M. (9 de febrero de 2015). ¿Por qué fracasan las pymes en Colombia? Dinero. Recuperado de https://bit.ly/3tqm7ls

Pradhan, R. K.; Jena, L. K.; Singh, S. K. (2017). Examining the role of emotional intelligence between organizational learning and adaptive performance in Indian manufacturing industries. Journal of Workplace Learning, 29(3), 235-247. DOI: https:// doi.org/10.1108/JWL-05-2016-0046

Priyono, A. (2016). The use of ICT platforms to promote knowledge exchange in project-based organisations. International Journal of Entrepreneurial Knowledge, 4(2), 5-21. DOI: https://doi.org/10.37335/ijek.v4i2.43

Qi, C.; Chau, P. Y. K. (2018). Will enterprise social networking systems promote knowledge management and organizational learning? An empirical study. Journal of Organizational Computing and Electronic Commerce, 28(1), 31-57. DOI: https://doi.org/10.1080/10919392.2018.1407081

Quintero R. (2019). La Mipyme, como impulsor de la economía nacional. Ponencia presentada en el $64^{\circ}$ Congreso Nacional Mipyme. Acopi, Barranquilla, Colombia, 29-30 de agosto.

Quiroga-Parra, D.; Vásquez-Rizo, F. E.; Montaño-Motato, H.; Espinosa-Correa, D.; Hernández-Arias, B. H.; GutiérrezMorales, P. A. (2010). La gestión del conocimiento y las tecnologías de la información y la comunicación en las organizaciones. Cali: Grupo de Investigación en Gestión del Conocimiento y Sociedad de la Información.

RAE (Real Academia Española). (28 de agosto de 2019). Conocimiento. Definición de conocimiento. Diccionario de la lengua española. Edición del Tricentenario. Recuperado de https://bit.ly/30NWX3R

Riascos-Erazo, S. C.; Aguilera-Castro, A. (2011). Herramientas TIC como apoyo a la gestión del talento humano. Cuadernos de Administración Universidad Del Valle, 27(46), 141-54. DOI: https://doi.org/10.25100/cdea.v27i46.88

Senge M. P. (1999). La quinta disciplina. Barcelona: Granica.

Soto-Acosta, P.; Cegarra-Navarro, J.-G. (2016). New ICTs for Knowledge Management in Organizations. Journal of Knowledge Management, 20(3), 417-422. DOI: https://doi. org/10.1108/JKM-02-2016-0057 
Stankosky, M. (2005). Creating the discipline of knowledge management. Oxford, UK: Elsevier Inc.

Vrontis, D.; Thrassou, A.; Santoro, G.; Papa, A. (2017). Ambidexterity, external knowledge and performance in knowledge-intensive firms. Journal of Technology Transfer, 42(2), 374-388. DOI: https://doi.org/10.1007/s10961-0169502-7

Weinzimmer, L. G.; Esken, C. A. (2017). Learning from mistakes: how mistake tolerance positively affects organizational learning and performance. Journal of Applied Behavioral Science, 53(3), 322-348. DOI: https://doi. org/10.1177/0021886316688658

Yunis, M.; Tarhini, A.; Kassar, A. (2018). The role of ICT and innovation in enhancing organizational performance: The catalysing effect of corporate entrepreneurship. Journal of Business Research, 88, 344-356. DOI: https://doi. org/10.1016/j.jbusres.2017.12.030 